\title{
Ultrasound-Guided Superficial Serratus Plane Block for Persistent Post-Mastectomy Pain: Four Case Reports
}

\section{Qian Liu ( $\nabla$ qianliu@cqmu.edu.cn )}

Institute of Neuroscience, Basic Medical College, Chongqing Medical University https://orcid.org/00000002-4485-744X

\section{Khanna Ashish}

Department of Cancer Rehabilitation, Winship Cancer Institute, Emory University School of Medicine

\section{Michael Stubblefield}

Department of Physical Medicine and Rehabilitation, Rutgers New Jersey Medical School, Select Medical, Kessler Institute of Rehabilitaion

\section{Guanghui Yue}

Centerfor Mobility and Rehabilitation Engineering Research, Kessler Foundation

\section{Didier Allexandre}

Center for Mobility and Rehabilitation Engineering Research, Kessler Foundation

\section{Research Article}

Keywords: Persistent post-mastectomy pain, Superficial serratus plane block, Novel regional anesthetic technique, Case report

Posted Date: August 13th, 2021

DOI: https://doi.org/10.21203/rs.3.rs-708986/v1

License: (9) (1) This work is licensed under a Creative Commons Attribution 4.0 International License. Read Full License

Version of Record: A version of this preprint was published at Supportive Care in Cancer on November 27th, 2021. See the published version at https://doi.org/10.1007/s00520-021-06696-7. 


\section{Abstract}

Background: Persistent post-mastectomy pain (PPMP) is common after surgery. Although multiple modalities have been used to treat this type of pain, including physical medicine, physical therapy and interventional approaches, managing PPMP may be still a challenge for breast cancer survivors. Currently, serratus plane block (SPB) as a novel regional anesthetic technique shows promising results for controlling chronic pain.

Methods: We report four cases of patients with PPMP that were performed using superficial serratus plane block (SSPB) at our clinic. A retrospective review of effect of pain relief was collected through postprocedure interviews.

Results: We found that two of our patients were successfully treated with SSPB for pain after treatment for breast cancer. The third patient had an intercostobrachial nerve block that produced incomplete pain relief, but had adequate pain relief with a SSPB. However, the fourth patient reported no pain relief after SSPB.

Conclusion: These cases illustrate that the patients with PPMP could benefit from SSPB. Particularly, we find patients with a subjective sense of "tightness" relating to reconstructive surgeries may be a good candidate for SSPB. Further studies are warranted to evaluate this block for PPMP, as it is low risk and relatively simple to perform.

\section{Introduction}

Breast cancer is the most common malignancy in women worldwide ${ }^{[1]}$.Thousands of patients undergo surgical procedures in the chest and axilla. Mastectomy with or without reconstruction remains a relatively common surgical procedure and can be associated with noticeable and chronic postoperative pain. The characterization of persistent post-mastectomy pain (PPMP) has been termed in recent studies, which include more sources of post-mastectomy pain, such as lymphedema and musculoskeletal pain. The definition of PPMP is broader than post-mastectomy pain syndrome ${ }^{[2]}$. Pain is typically localized to the axillary region, breast, chest wall, and medial upper extremity and described as "Shooting", "stabbing", "pulling", "tightness", "burning" and "aching" [2]. It has been described that PPMP occurs in about as many as $25-60 \%$ of patients ${ }^{[3,4]}$. Almost $30 \%$ of breast cancer survivors experience above-average pain up to 10 years after treatment ends ${ }^{[5]}$. Abundant evidence demonstrates that pain negatively impacts sleep, activities of daily living, social interactions, and overall quality of life ${ }^{[6]}$. Advances in treatment have increased five-year survival rates and longevity ${ }^{[7]}$. As a result, people are living longer with their chronic pain. This increase in survivorship underscores the importance of managing pain for improving quality of life for breast cancer survivors.

Currently, pain management of PPMP has involved multiple modalities including physical medicine and rehabilitation, pharmacological therapy, physical therapy, exercise interventions, cognitive-behavioral 
psychology, psychosocial interventions, and interventional approaches. Survivors suffering from PPMP who have insufficient pain control or significant adverse side effects from pharmacotherapy may be amenable to interventional approaches. In terms of interventional procedures, the neural blockade directed at the stellate ganglion, paravertebral plexus, and intercostal nerves have all been utilized with varying degrees of success ${ }^{[8]}$. Another alternative for an interventional procedure for PPMP is the serratus plane block (SPB).

The SPB is performed in the axillary region. First described in detail by Blanco et al in $2013^{[9]}$, SPB is a novel, ultrasound-guided regional anesthetic technique. Two potential spaces were identified: one superficial and another deep to the serratus anterior muscle at the level of fifth rib in the mid-axillary line were identified $^{[9]}$. The superficial serratus anterior plane is a fascial space that is created between the posterior surface of the latissimus dorsi and the most anterior surface of the serratus anterior muscle. The deep serratus anterior plane exists between the serratus anterior muscle and external intercostal muscle at approximately the level of the fifth and sixth ribs ${ }^{[9,10]}$. Currently, SPB has been employed as an effective and safe means for persistent pain after breast cancer surgery or postmastectomy pain syndrome $^{[11,12]}$. The research about the effect of superficial serratus plane block (SSPB) or deep serratus plane block (DSPB) for managing pain produced conflicting conclusions. Blanco et al suggest the SSPB showed more promise in terms of anesthetic spread and sensory distribution ${ }^{[9]}$. Another study suggest that the DSPB is associated with early postoperative analgesia that is as effective as the SSPB following breast cancer surgery ${ }^{[13]}$. Piracha and colleagues demonstrate that the DSPB can provide longer-duration pain relief for postmastectomy pain syndrome than SSPB ${ }^{[10]}$. However, the DSPB may be difficult to perform effectively ${ }^{[10]}$. The superficial serratus plane is easier to identify, making SSPB a much simpler and safer block ${ }^{[9]}$. Anatomical study revealed that the extent of injectate spread was mostly influenced by the volume of injection rather than the plane of injection relative to the serratus anterior muscle ${ }^{[14]}$. As a result, we would prefer to use SSPB for managing pain of patients after breast cancer surgery. This article presents a case series of the application and efficacy of the SSPB for the patients with PPMP.

\section{Treatment Intervention}

The retrospective chart review approved by the institutional review board at the Kessler Institute for Rehabilitation was performed for four patients with PMPS who underwent treatment with the SSPB. Risks and benefits were discussed with the patient prior to the procedure. To perform the block, patients were positioned in the lateral decubitus position with the arm moved forward into extension. The operator was seated behind the patient with the ultrasound display in front of the patient(Fig. 1). This allows for a clear line of sight of the area of the injection and the ultrasound screen. After sterile preparation, sagittal images of the latissimus dorsi and serratus anterior muscle were identified by placing a linear ultrasound probe at the midaxillary line (Fig. 2). The block was performed by first identifying the muscle layers of the latissimus dorsi muscle and the serratus anterior muscle using a SonoSite Edge II ultrasound machine with a $15-6$ megahertz probe (Fujifilm, Bothell, Washington) and sterile gel. Once the fascial layers were identified, a total of $3 \mathrm{~mL}$ of $0.25 \%$ bupivacaine, $6 \mathrm{~mL}$ of $1 \%$ lidocaine with $1 \mathrm{~mL}$ of $40 \mathrm{mg} / \mathrm{ml}$ of 
triamcinolone was injected under direct ultrasound visualization, and adequate spread was noted between the serratus anterior muscle and the latissimus dorsi muscle in real time (Fig. 3).

\section{Results}

\section{Patient 1}

A 68-year-old woman with history of right-sided breast intraductal carcinoma, status post right mastectomy and prophylactic left mastectomy, presented with severe pain described as "burning" and "tightness" "deep inside" the bilateral chest wall for the past 9 years. Her pain was uncontrolled with Lyrica and Valtrex. Lidocaine patches were not effective. The decision was made to trial SSPB as an appropriate treatment for her bilateral PMPS. We repeated the procedure 7 and then 14 days later. After the first injection, the patient reported that her pain had decreased about 10-15\%. A second block was performed one week later, further reducing her pain about $50 \%$. After the third block, she reported $97 \%$ pain relief. At 4-week follow-up, near-full pain relief continued. At 4-months follow-up, patient reported almost $90 \%$ pain relief was maintained. With improved pain management, she was better able to resume her daily activities.

\section{Patient 2}

A 53-year-old woman presented to our clinic with severe breast pain as well as axillary and anterolateral chest wall pain. Her breast carcinoma was treated with a double mastectomy, placement of tissue expanders and bilateral breast implants. She described the pain as "soreness" and "tightness" that started following tissue expander placement four years earlier. She rated her pain 10/10 on a numerical pain scale at worst.

She had tried Voltaren gel, Metaxalone, Etodolac, Tylenol Extra Strength, IcyHot with lidocaine and Celebrex with no relief. After discussion, it was determined that SSBP would be an appropriate intervention to try as an effective modality to aid in relief of pain. We repeated the SSPB twice more, 3 weeks after the first injection and then again 4 weeks later. The patient stated that after the first injection, she had had $80 \%$ improvement in her feeling of "tightness". After the second block, she reported that she felt $90 \%$ relief. After the third block, she reported $100 \%$ pain relief. This pain relief persisted at 4 months.

\section{Patient 3}

A 64-year-old woman with a history of right-sided breast cancer, who was status post mastectomy and axillary node dissection followed by reconstruction, presented with right-sided breast pain of three years duration. Pain was particularly intense at the tail of the breast at the chest wall near the axilla. She described the pain as "soreness" and "jabbing and stabbing" when she moves or rotates her thorax. Maximal area of pain was in the distribution of the intercostobrachial nerve, a cutaneous sensory branch of the second intercostal nerve innervating the lateral chest wall, axilla, and posterior portion of the proximal arm. This nerve is frequently sacrificed in axillary node dissections. The patient was taking 
Tramadol 1-2 times daily for pain. Firstly, the decision was made to perform an intercostobrachial nerve block to address her primary pain generator. The nerve block under ultrasound guidance with $1 \mathrm{ml}$ of $40 \mathrm{mg} / \mathrm{ml}$ triamcinolone and $4 \mathrm{ml}$ of $1 \%$ lidocaine. It provided $80 \%$ relief of stabbing pains. Afterwards, the patient had a new complaint of "tightness" diffusely. After discussion, the decision was made to perform SSPB as well. We repeated the SSPB 7 and then 14 days later. After the first SSPB block, the patient reported $85 \%$ improvement. After the second and third block, she reported that she had $90 \%$ pain relief. The feeling of "stabbing" pain relief lasted for the next 2 months. Other noted improvements were decreased neck pain and increased pain-free range of motion of the right shoulder.

\section{Patient 4}

A 54-year-old woman had history of right ductal carcinoma in situ about three years prior to her initial clinic visit. She had undergone lumpectomy shortly after the diagnosis but positive margins necessitated that she return to the operating room for bilateral nipple-sparing mastectomies with bilateral sentinel lymph node biopsies. She had immediate reconstruction with tissue expanders. However, definitive implant placement six months later was prepectoral as opposed to behind the pectoralis muscle. She described bilateral chest wall "spasms" that began approximately two weeks post-operatively that had persisted. The spams quickly degenerated into severe neuropathic pain bilaterally, with a "squeezing" feeling described "as if someone were standing on my chest." Over the years she had tried physical therapy with myofascial release, gabapentin, and duloxetine with either no relief or limiting side effects. She had no improvement following a number of procedures including intercostal nerve blocks, pulsed radiofrequency ablation, and botulinum toxin injections to the chest wall and intercostals. She then agreed to a trial of SSPB to address the tightness feeling, given the increased suspicion that the tightness may be of neuropathic origin. She reported no relief of tightness after the first injection but did report a small reduction in other neuropathic pain symptoms of "burning" and "stabbing". Following the second injection two week later she felt no further relief of any symptoms and elected not to return for the third in the series and was subsequently lost to follow-up.

\section{Discussion}

While SPB is effectively used perioperatively for breast and thoracic wall surgeries, this block reportedly provides satisfactory pain relief for persistent post surgical pain even years later, as some case reports have recently described ${ }^{[11,15]}$. However, pain relief was inconsistent in these case reports. It is worth investigating why the SPB works for only some patients, rather than for all. We describe 3 of 4 cases in which the SSPB provided good efficacy in improved pain relief in breast cancer patients with PPMP. In each case, the SSPB procedure was performed safely and without any complications.

Patient 1 presented severe pain throughout the chest wall after bilateral mastectomies and received significant pain relief from bilateral SSPB. Patient 2 presented with severe breast pain, axillary and anterolateral chest wall tightness status post mastectomy, placement of tissue expanders and eventual bilateral breast implants. The SSPB block resulted in apparent pain relief. Patient 3 presented with breast 
pain status post mastectomy, lymph node dissection and reconstruction. The patient received reasonable relief of "jabbing and stabbing" pain from an intercostobrachial nerve block. However, once this pain was ameliorated, tightness became the primary complaint and she then received further significant relief from a SSPB.

It is of particular interest that we found that these three patients had in common a complaint of "tightness" among other descriptors. Two of the patients had breast reconstruction with implants and/or tissue expanders. Roth et al reported that women who undergo reconstructive surgery with implants appear to be at higher risk than those without implants for breast pain ${ }^{[16]}$. Tightness is one of most frequently used descriptors by patients with PPMP, particularly in the reconstructed breast. Zocca et al. reported on eight patients undergoing SPB, four of whom described their pain as "tightness" or "pressure" and six of whom had reconstruction. ${ }^{[11]}$.

A leading theory is based on the fact that breast tissue is embedded in the fascial web of connective tissue which runs continuously throughout the body. Anatomically, there are nerves traversing the fascial planes and others that travel within the fascia. We hypothesize that the breast implants may press against this fascial web of the chest and irritate the nerves, nerve branches, or nerve endings within the chest when the implants were placed under the muscle intraoperatively.

Recent research shows a direct link between inflammation of the fascia and pain. ${ }^{[17,18]}$ The use of corticosteroid injected in the interfacial planes could have contributed to tempering this continuous inflammatory condition ${ }^{[15]}$. The addition of dexamethasone to local anesthetic in a nerve block has been reported to prolong the duration of the nerve block ${ }^{[19,20]}$. Triamcinolone is a popular corticosteroid used for increasing the duration of action of local anesthetics ${ }^{[21]}$. In our cases, the use of triamcinolone in conjunction with local anesthetic might have decreased the nociceptive component of pain.

As seen in this case series, we did have some good outcomes, however, the effect of the SSPB was not successful in all postmastectomy patients. In the fourth case, no obvious improvement was seen by using SSPB in a patient who also had persistent pain after mastectomy, lymph node dissection and reconstruction. Similarly, Piracha et al and Zocca et al ${ }^{[10,11]}$ also observed that a subset of patients did not have any appreciable improvement in their pain. In their opinion, the presence of iatrogenic scar tissue, whether from radiation or surgery, may prevent the necessary separation of the fascial planes required for adequate spread of the injectate ${ }^{[10,11]}$. Additionally, we further speculate that the fascia may become thicker and less pliable around the area of scarring due to relative muscle immobility. This increased fascial thickness could lead to insufficient spread of the injectate along the superficial plane.

There are some differing conclusions as to the mechanism of action in the SPB. Clinically, results showed that SPB anaesthetizes the lateral cutaneous branches of the thoracic intercostals nerves II to VI, the long thoracic nerve and the thoracodorsal nerve ${ }^{[9,10]}$. However, although the long thoracic nerve and thoracodorsal nerve lie on the surface of the serratus anterior muscle, anatomical study showed that serratus plane block appears to function through blockade of the lateral cutaneous branches of the 
intercostal nerves, rather than through direct block of the intercostal nerves, the long thoracic nerve and thoracodorsal nerve ${ }^{[22]}$. Another anatomical study also showed a narrower range of spread of colored dye even larger volumes of injectate ${ }^{[23]}$. The reason of this difference may be the gap between anatomical evaluation of range of spread and clinical practice. Lastly, another mechanism of pain relief may be the motor block of pectoral and serratus muscles, allowing the extra volume of an implant to be accommodated.

We feel these cases underscore some findings that may be helpful as a guide in patient selection. Based on our experience, we found the SSPB to be relatively successful for pain relief of those with a primary complaint of tightness over the breast or anterior and lateral chest wall, both in patients with or without implants. In particular, women with pain described as tightness as a result of reconstructive surgery with implant placement may be even more ideal candidates for SSPB.

Our results suggest that SSPB is a safe block suitable to be done in the outpatient setting. It is a promising method for reducing levels of pain experienced by patients with PMPS. However, this study is limited in that it is a descriptive study and further comparative randomized controlled clinical trials will be needed to further validate this block.

\section{Declarations}

Funding: N/A

Conflicts of interest/Competing interests: The authors declare that they have no conflict of interest. The authors have full control of all primary data and agree to allow the journal to review the data if requested.

Availability of data and material: The datasets used are available from the corresponding author on reasonable request.

Code availability: N/A.

Authors' contributions: Dr.Liu designed the study, included the participants in the study and wrote the first draft of the manuscript. Dr.Ashish contributed in diagnosing the disease, treating the patients and overall coordination with regard to the manuscript. He also corrected and revised the manuscript. Dr.Stubblefield is a consultant physician of the clinic where the patient was treated. Contributed in the clinical diagnosis of the patient's condition and doing the required referrals to confirm the diagnosis. He also designed the study and revised the manuscript. Dr.Yue and Allexandre designed the study and prepared the files for the INSTITUTIONAL REVIEW BOARD (IRB) of Kessler Institute for Rehabilitation. They also revised the manuscript. All authors contributed to the article and approved the submitted version.

Ethics approval: This case report is a chart review study of patients on which we have done the nerve block. We only recorded the information from the charts in a manner that cannot identify the subjects 
either directly or indirectly. The INSTITUTIONAL REVIEW BOARD (IRB) of Kessler Institute for Rehabilitation responded to us that the study is exempt from IRB review.

Consent to participate: Written informed consent was obtained from the patient for publication of this manuscript and any accompanying images.

Consent for publication: Informed written consent for publishing the patient's details in the journal was obtained and is available for the perusal of the editors upon request.

\section{References}

1. Ferlay J, Soerjomataram I, Dikshit R, Eser S, Mathers C, Rebelo M, et al. Cancer incidence and mortality worldwide: sources, methods and major patterns in GLOBOCAN 2012. International journal of cancer 2015; 136(5):E359-386.

2. Tait RC, Zoberi K, Ferguson M, Levenhagen K, Luebbert RA, Rowland K, et al. Persistent PostMastectomy Pain: Risk Factors and Current Approaches to Treatment. The journal of pain : official journal of the American Pain Society 2018; 19(12):1367-1383.

3. Belfer I, Schreiber KL, Shaffer JR, Shnol H, Blaney K, Morando A, et al. Persistent postmastectomy pain in breast cancer survivors: analysis of clinical, demographic, and psychosocial factors. The journal of pain : official journal of the American Pain Society 2013; 14(10):1185-1195.

4. Andersen KG, Kehlet $\mathrm{H}$. Persistent pain after breast cancer treatment: a critical review of risk factors and strategies for prevention. The journal of pain : official journal of the American Pain Society 2011; 12(7):725-746.

5. Forsythe LP, Alfano CM, George SM, McTiernan A, Baumgartner KB, Bernstein L, et al. Pain in longterm breast cancer survivors: the role of body mass index, physical activity, and sedentary behavior. Breast cancer research and treatment 2013; 137(2):617-630.

6. Ancoli-Israel S, Liu L, Rissling M, Natarajan L, Neikrug AB, Palmer BW, et al. Sleep, fatigue, depression, and circadian activity rhythms in women with breast cancer before and after treatment: a 1-year longitudinal study. Supportive care in cancer : official journal of the Multinational Association of Supportive Care in Cancer 2014; 22(9):2535-2545.

7. DeSantis CE, Ma J, Goding Sauer A, Newman LA, Jemal A. Breast cancer statistics, 2017, racial disparity in mortality by state. CA: a cancer journal for clinicians 2017; 67(6):439-448.

8. Wijayasinghe N, Andersen KG, Kehlet $\mathrm{H}$. Neural blockade for persistent pain after breast cancer surgery. Regional anesthesia and pain medicine 2014; 39(4):272-278.

9. Blanco R, Parras T, McDonnell JG, Prats-Galino A. Serratus plane block: a novel ultrasound-guided thoracic wall nerve block. Anaesthesia 2013; 68(11):1107-1113.

10. Piracha MM, Thorp SL, Puttanniah V, Gulati A. "A Tale of Two Planes": Deep Versus Superficial Serratus Plane Block for Postmastectomy Pain Syndrome. Regional anesthesia and pain medicine 2017; 42(2):259-262. 
11. Zocca JA, Chen GH, Puttanniah VG, Hung JC, Gulati A. Ultrasound-Guided Serratus Plane Block for Treatment of Postmastectomy Pain Syndromes in Breast Cancer Patients: A Case Series. Pain practice : the official journal of World Institute of Pain 2017; 17(1):141-146.

12. Takimoto K, Nishijima K, Ono M. Serratus Plane Block for Persistent Pain after Partial Mastectomy and Axillary Node Dissection. Pain physician 2016; 19(3):E481-486.

13. Abdallah FW, Cil T, MacLean D, Madjdpour C, Escallon J, Semple J, et al. Too Deep or Not Too Deep?: A Propensity-Matched Comparison of the Analgesic Effects of a Superficial Versus Deep Serratus Fascial Plane Block for Ambulatory Breast Cancer Surgery. Regional anesthesia and pain medicine 2018; 43(5):480-487.

14. Biswas A, Castanov V, Li Z, Perlas A, Kruisselbrink R, Agur A, et al. Serratus Plane Block: A Cadaveric Study to Evaluate Optimal Injectate Spread. Regional anesthesia and pain medicine 2018; 43(8):854858.

15. Wijayasinghe N, Duriaud HM, Kehlet $H$, Andersen KG. Ultrasound Guided Intercostobrachial Nerve Blockade in Patients with Persistent Pain after Breast Cancer Surgery: A Pilot Study. Pain physician 2016; 19(2):E309-318.

16. Roth RS, Lowery JC, Davis J, Wilkins EG. Persistent pain following postmastectomy breast reconstruction: long-term effects of type and timing of surgery. Annals of plastic surgery 2007; 58(4):371-376.

17. Hoheisel $U$, Rosner J, Mense S. Innervation changes induced by inflammation of the rat thoracolumbar fascia. Neuroscience 2015; 300:351-359.

18. Wilke J, Schleip R, Klingler W, Stecco C. The Lumbodorsal Fascia as a Potential Source of Low Back Pain: A Narrative Review. BioMed research internationa/ 2017; 2017:5349620.

19. Movafegh A, Razazian M, Hajimaohamadi F, Meysamie A. Dexamethasone added to lidocaine prolongs axillary brachial plexus blockade. Anesthesia and analgesia 2006; 102(1):263-267.

20. Parrington SJ, O'Donnell D, Chan VW, Brown-Shreves D, Subramanyam R, Qu M, et al. Dexamethasone added to mepivacaine prolongs the duration of analgesia after supraclavicular brachial plexus blockade. Regional anesthesia and pain medicine 2010; 35(5):422-426.

21. Kim HS, Lee SJ, Kim CS, Kim JT. Effect of triamcinolone added to scalp nerve block for postoperative pain management of Moyamoya disease. Korean journal of anesthesiology 2011; 61(2):173-174.

22. Mayes J, Davison E, Panahi P, Patten D, Eljelani F, Womack J, et al. An anatomical evaluation of the serratus anterior plane block. Anaesthesia 2016; 71(9):1064-1069.

23. Kunigo T, Murouchi T, Yamamoto S, Yamakage M. Spread of injectate in ultrasound-guided serratus plane block: a cadaveric study. JA clinical reports 2018; 4(1):10.

\section{Figures}




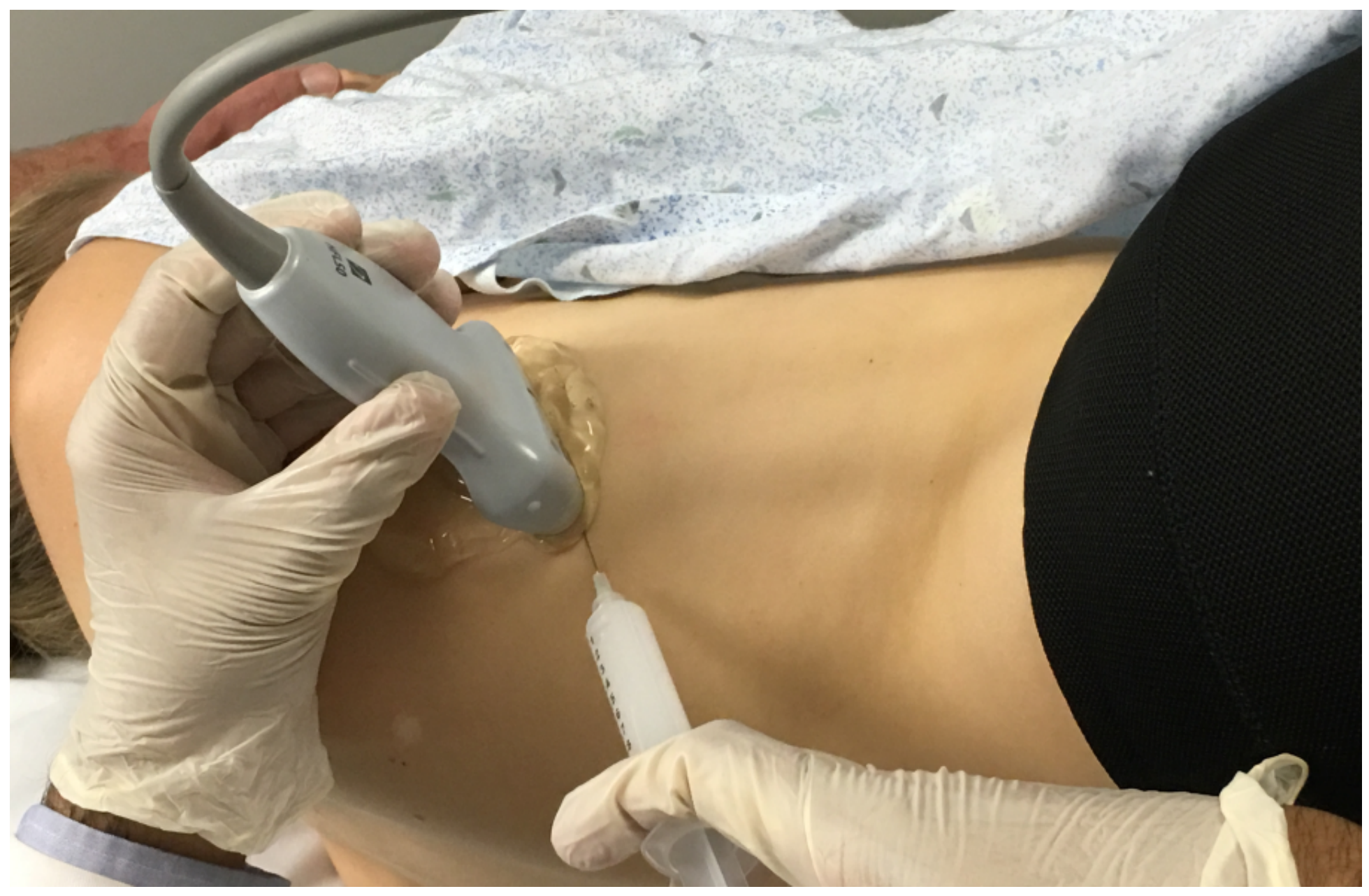

Figure 1

The position of the ultrasound transducer and injection needle. The ultrasound probe was positioned perpendicular to the thoracic wall. The needle is inserted in a caudad to cephalad direction to the muscular plane.

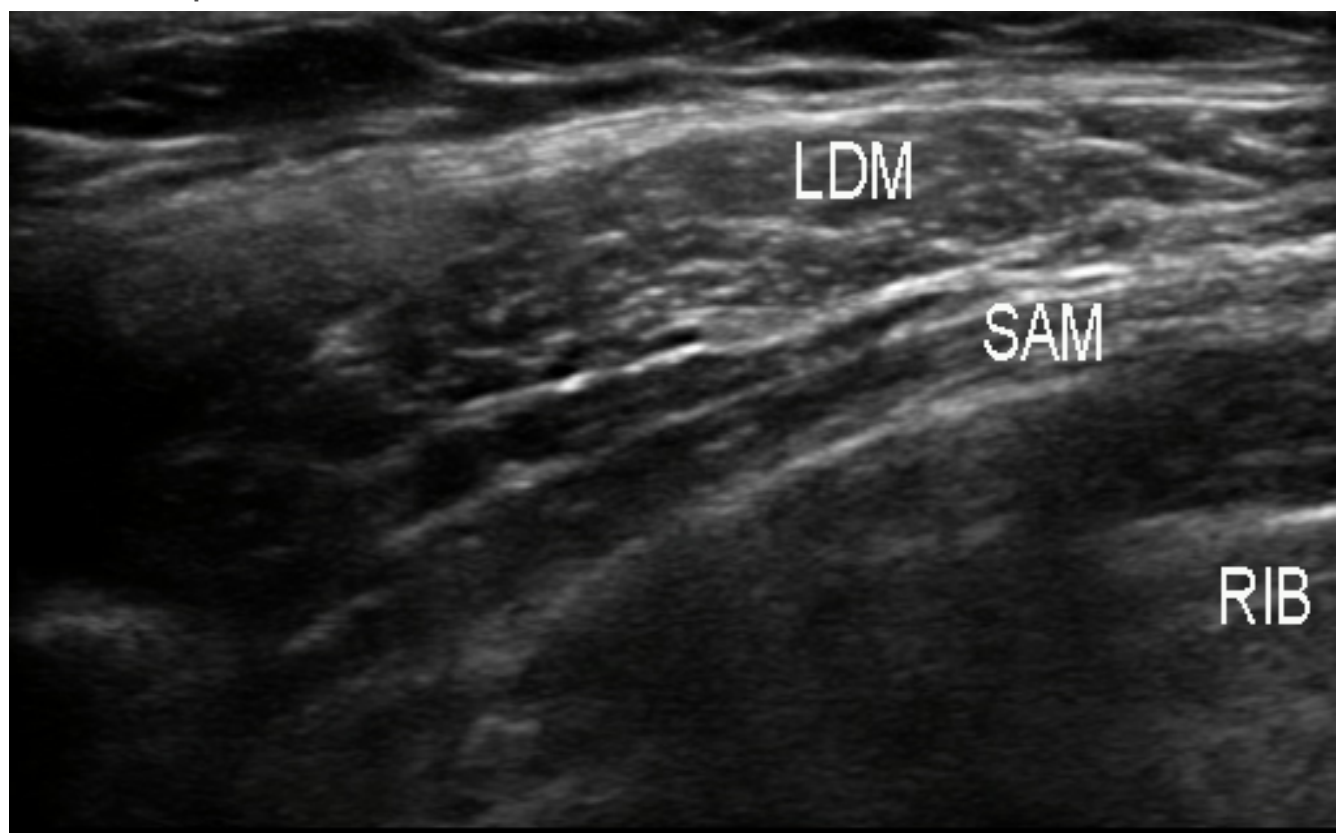


Figure 2

Sonographic images prior to block. Planes identified. LDM indicates latissimus dorsi muscle; SAM, serratus anterior muscle; RIB, fifth rib.

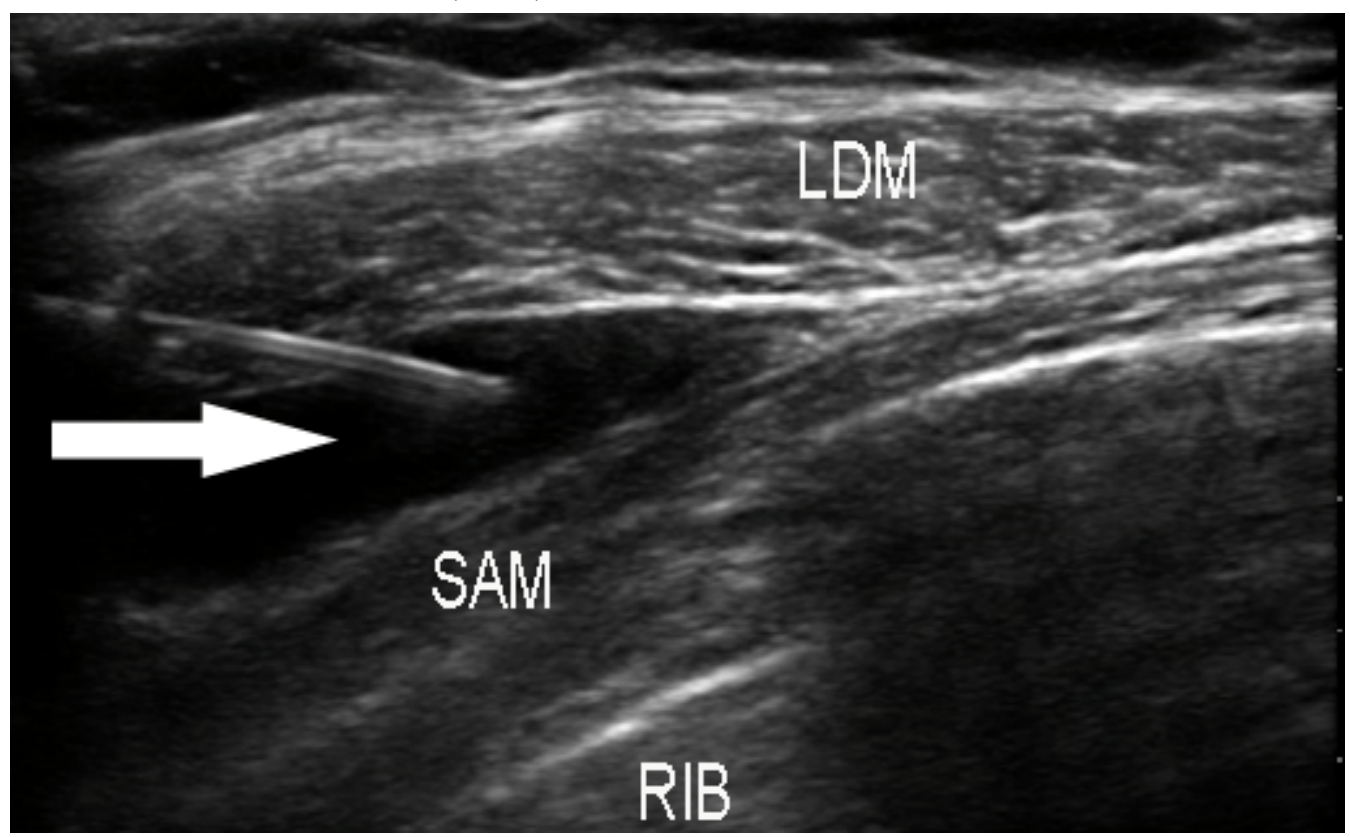

\section{Figure 3}

Sonographic images when the planes above the serratus muscle are dissected using local anaesthetic. LDM indicates latissimus dorsi muscle; SAM, serratus anterior muscle; RIB, fifth rib; solid arrow = spread of local anesthetic. 